\title{
THE MOMENTS OF THE NUMBER OF CROSSINGS OF A LEVEL BY A STATIONARY NORMAL PROCESS
}

\author{
By Harald Cramér and M. R. Leadbetter ${ }^{1}$ \\ Stockholm University and Research Triangle Institute
}

0. Summary. In this paper we consider the number $N$ of upcrossings of a levt $u$ by a stationary normal process $\xi(t)$ in $0 \leqq t \leqq T$. A formula is obtained for the factorial moment $M_{k}=\mathcal{E}\{N(N-1) \cdots(N-k+1)\}$ of any desired order $k$. The main condition assumed in the derivation is that $\xi(t)$ have, with probability one, a continuous sample derivative $\xi^{\prime}(t)$ in the interval $[0, T]$. This condition involves hardly any restriction since an example shows that even a slight relaxation of it causes all moments of order greater than one to become infinite. The moments of the number of downcrossings or total number of crossings can be obtained analogously.

1. Introduction. The problem of obtaining the mean number of crossings, (or equivalently upcrossings) of a given level, by a stationary normal process in a given time, has received a good deal of attention in the literature. In fact, a complete solution to this problem has now been given by Ylvisaker [8]. However, moments of order greater than one of the number of crossings of a level have received less attention. The variance was obtained by Steinberg et al. [6], using somewhat heuristic arguments. Rozanov and Volkonski [7] point out in a footnote that the formula given in [6] for the variance is valid under certain precise conditions, of which the main one is that the covariance function of the process have a finite sixth derivative at the origin. Finally in this connection, the variance has been obtained by Leadbetter and Cryer [4] under conditions which assume just a little more than the existence of a second derivative of the covariance function.

There is virtually no literature available in connection with moments of the number of crossings of a level, of higher order than the second. (A partial result is indicated by Ivanov at the end of his paper [3].) It will be our purpose here to obtain explicit expressions for such moments. From conversations with Dr. $\mathrm{Yu}$. K. Belayev, we know that he has investigated certain problems related to the asymptotic behaviour of the moments of the number of crossings. The explicit expression for these moments which we prove seems, however, to be new.

Our treatment in the sequel will be in terms of upcrossings and we shall obtain explicit expressions for factorial moments of arbitrary orders, under conditions which are very close to the necessary ones. Corresponding formulae for moments of all orders for the downcrossings, or total number of crossings, follow similarly.

2. Moments of the number of upcrossings. We shall, throughout, consider a

Received 8 March 1965.

${ }^{1}$ Research was supported in part by the Office of Naval Research. 
real valued stationary normal process $\{\xi(t): 0 \leqq t \leqq T\}$ having (for convenience) zero mean, spectrum $F(\lambda)$ possessing a continuous component, and covariance function $r(\tau)=\int_{-\infty}^{\infty} e^{i \lambda \tau} d F(\lambda)$. We shall further assume that $\xi(t)$ has, with probability one, a continuous sample derivative $\xi^{\prime}(t)$ on the interval $[0, T]$. Sufficient conditions for this latter property in terms of the behaviour of the covariance function are given, for example, in [1]. Write $N$ for the number of $u p$ crossings of the level $u$ by $\xi(t)$ in $0 \leqq t \leqq T$; that is $N$ is the number of points $t$ in that interval for which $\xi(t)=u, \xi^{\prime}(t)>0$. Then the following result holds.

Theorem. If $\{\xi(t): 0 \leqq t \leqq T\}$ is a normal stationary process, as described, possessing, with probability one, a continuous sample derivative, and $k$ is any positive integer, then

$$
\begin{aligned}
M_{k} & =\mathcal{E}\{N(N-1) \cdots(N-k+1)\} \\
& =\int_{0}^{T} \cdots \int_{0}^{T} d t_{1} \cdots d t_{k} \int_{0}^{\infty} \cdots \int_{0}^{\infty} y_{1} \cdots y_{k} p_{\mathfrak{t}}(u, \mathrm{y}) d y_{1} \cdots d y_{k}
\end{aligned}
$$

in which $p_{\mathfrak{t}}(u, \mathbf{y})=p_{\mathfrak{t}}\left(u, \cdots, u, y_{1}, \cdots, y_{k}\right), p_{\mathfrak{t}}\left(x_{1}, \cdots, x_{k}, y_{1}, \cdots, y_{k}\right) d e-$ noting the joint density for the random variables $\xi\left(t_{1}\right) \cdots \xi\left(t_{k}\right), \xi^{\prime}\left(t_{1}\right) \cdots \xi^{\prime}\left(t_{k}\right)$.

We note here that it follows from the appendix that, when all $t_{i}$ are different, $p_{\mathrm{t}}$ is the density corresponding to a non singular joint distribution since $F(\lambda)$ is assumed to have a continuous component.

Before proceeding to the proof we note that the theorem can easily be modified to refer to "downcrossings" or the total number of crossings of the level $u$ in time $T$. The discussion will be given here in terms of upcrossings, however.

The following proof is divided into two parts A and B. In Part A it is shown that $M_{k}$ does not exceed the expression on the right hand side of (1), whereas in Part B the reverse inequality is proved. The techniques are straightforward but quite different in each part. It is a perhaps somewhat surprising feature, however, that in both parts use can be made of Fatou's lemma to give the essential inversions of limiting operations with integrations, in order that inequalities in the desired (opposite) directions may be obtained.

Proof of THE Theorem.

Part A. Write $\xi(t)=\xi(t, \omega)$ to exhibit explicit dependence on the "sample point" $\omega \varepsilon \Omega$. Let $S$ denote the set of all $\omega$ such that $\xi^{\prime}(t)$ is continuous in the interval $I=[0, T]$, while the equation $\xi(t)=u$ has at most a finite number of roots $t$ in $I$, and while further $\xi(0) \neq u \neq \xi(T)$ and $\xi^{\prime}(t) \neq 0$ whenever $\xi(t)=u$. According to Bulinskaya [2], Theorem 1, we then have

$$
P(S)=1 \text {. }
$$

Write now $(N)_{k}=N(N-1) \cdots(N \perp k+1)$ for $k=1,2, \cdots$ and define the functions $\delta_{n}(x), \sigma(x)$ by

$$
\begin{aligned}
\delta_{n}(x) & =n, & & |x| \leqq 1 /(2 n) \\
& =0, & & \text { otherwise }
\end{aligned}
$$

and

$$
\begin{aligned}
\sigma(x) & =x, & & x>0, \\
& =0, & & \text { otherwise. }
\end{aligned}
$$


Let $D(\epsilon)$ denote the domain in the $k$-dimensional space $R^{k}$ with coordinates $t_{1} \cdots t_{k}$ defined by the inequalities

$$
\begin{aligned}
0<t_{i}<T & \text { for } i=1 \cdots k, \\
\left|t_{i}-t_{j}\right|>\epsilon & \text { for } i \neq j .
\end{aligned}
$$

Define also the random variable $J_{k}(n, \epsilon, \omega)$ by the relation

$$
J_{k}(n, \epsilon, \omega)=\int \cdots \int_{D(\epsilon)} \prod_{i=1}^{k}\left\{\delta_{n}\left[\xi\left(t_{i}\right)-u\right] \sigma\left[\xi^{\prime}\left(t_{i}\right)\right]\right\} d t_{1} \cdots d t_{k} .
$$

We shall. now proceed to prove that

$$
\mathcal{E}\left\{(N)_{k}\right\} \leqq \lim _{\epsilon \rightarrow 0} \lim _{n \rightarrow \infty} \varepsilon\left\{J_{k}(n, \epsilon, \omega)\right\} .
$$

In order to prove the validity of (4) we define a subset $S(h)$ of $S$ consisting of all $\omega \varepsilon S$ for which the following two conditions are satisfied:

(a) the distance between any two upcrossings in $I$ by $\xi(t)$ of the level $u$ is greater than $2 h$,

(b) for any zero $t=t_{0}$ of the derivative $\xi^{\prime}(t)$ in $I$, we have $\left|\xi\left(t_{0}\right)-u\right|>h$.

According to the definition of $S$ every $\omega \varepsilon S$ must also belong to $S(h)$ for some $h>0$ so that

$$
S(h) \uparrow S \text { as } h \downarrow 0 .
$$

Take now any fixed $\omega \varepsilon S(h)$, and let $t=r_{1}, \cdots, r_{N}$ be all the upcrossings of the corresponding $\xi(t)=\xi(t, \omega)$ in $I$. Consider the $k$-dimensional interval $I^{k}$ in the space $R^{k}$, and let $A_{j_{1}, \cdots, j_{k}}$ denote the point in $I^{k}$ with coordinates $t_{1}=r_{j_{1}}$, $\cdots, t_{k}=r_{j_{k}}$, where each $j_{i}$ may assume the values $1,2, \cdots, N$. Clearly there are $N^{k}$ different points $A$, and among these there are exactly $(N)_{k}$ points $A^{\prime}$ such that no two of the $j_{i}$ are equal. Since $\omega \varepsilon S(h)$, these points $A^{\prime}$ will all be situated in the domain $D(2 h)$, while the remaining $N^{k}-(N)_{k}$ points $A$ will fall outside $D(2 h)$, and even outside $D(\epsilon)$, for any $\epsilon>0$.

Considering, still the same fixed $\omega \varepsilon S(h)$ we now take $n$ and $\epsilon$ such that $0<n^{-1}<\epsilon<h$ and consider the integral $J_{k}(n, \epsilon, \omega)$ defined by (3). The contribution to $J_{k}(n, \epsilon, \omega)$ arising from small disjoint $k$ dimensional blocks about each point $A^{\prime}$ is easily seen to be just $(N)_{k}$ for all sufficiently large $n$ (i.e. a unit contribution from each such block). The contribution from the remaining region is zero for all sufficiently large $n$. (This can be seen clearly from a picture by taking $k=2$ and writing down the integrals involved.) Hence for any fixed $\epsilon<h$, we can always find $n_{0}$ so large that, for all $n>n_{0}$ we have

$$
J_{k}(n, \epsilon, \omega)=(N)_{k},
$$

and hence also

$$
(N)_{k}=\lim _{n \rightarrow \infty} J_{k}(n, \epsilon, \omega) .
$$

Since this holds for any $\epsilon<h$, while the first member is independent of $\epsilon$, it follows that

$$
(N)_{k}=\lim _{\epsilon \rightarrow 0} \lim _{n \rightarrow \infty} J_{k}(n, \epsilon, \omega)
$$


for any fixed $\omega \varepsilon S(h)$. But $h$ can be chosen arbitrarily small and since $S(h) \uparrow S$ as $h \downarrow 0$ it follows that (6) holds for any $\omega \varepsilon S$, i.e. with probability one. Finally an application of Fatou's lemma to the $\epsilon$ and $n$-limits yields the result (4). Thus from (4) we obtain

$$
\begin{aligned}
& \mathcal{E}\left\{(N)_{k}\right\} \leqq \lim _{\epsilon \rightarrow 0} \lim _{n \rightarrow \infty} \int \cdots \int_{D(\epsilon)} d t_{1} \cdots d t_{k} \\
& {\left[n^{k} \int_{u-(2 n)^{-1}}^{u+(2 n)-1} \cdots \int_{u-(2 n)-1}^{u+(2 n)-1} d x_{1} \cdots d x_{k} \int_{0}^{\infty} \cdots \int_{0}^{\infty} y_{1} \cdots y_{k}\right.} \\
&\left.p_{\mathrm{t}}\left(x_{1} \cdots x_{k}, y_{1} \cdots y_{k}\right) d y_{1} \cdots d y_{k}\right] .
\end{aligned}
$$

The entire expression in square brackets on the right hand side of (7) clearly converges to $\int_{0}^{\infty} \cdots \int_{0}^{\infty} y_{1} \cdots y_{k} p_{\mathbf{t}}(u, \mathbf{y}) d y_{1} \cdots d y_{k}$. Further, it can be readily shown that this expression is bounded for all $t_{1} \cdots t_{k}$ in the region $D(\epsilon)$ (using the fact that the determinant of the covariance matrix of $\xi\left(t_{1}\right) \cdots \xi\left(t_{k}\right)$, $\xi^{\prime}\left(t_{1}\right) \cdots \xi^{\prime}\left(t_{k}\right)$ is bounded away from zero). Hence by dominated convergence

$$
\begin{aligned}
& \mathcal{E}\left\{(N)_{k}\right\} \\
& \quad \leqq \lim _{\epsilon \rightarrow 0} \int \cdots \int_{D(\epsilon)} d t_{1} \cdots d t_{k} \int_{0}^{\infty} \cdots \int_{0}^{\infty} y_{1} \cdots y_{k} p_{\mathbf{t}}(u, \mathbf{y}) d y_{1} \cdots d y_{k} .
\end{aligned}
$$

Finally by monotone convergence it follows that

$$
\mathcal{E}\left\{(N)_{k}\right\} \leqq \int_{0}^{T} \cdots \int_{0}^{T} d t_{1} \cdots d t_{k} \int_{0}^{\infty} \cdots \int_{0}^{\infty} y_{1} \cdots y_{k} p_{\mathrm{t}}(u, \mathbf{y}) d y_{1} \cdots d y_{k} .
$$

Part B. In order to prove the reverse inequality to (9) we adopt a different procedure (due to Ylvisaker [8]) for counting the number of upcrossings by $\xi(t)$ in $0 \leqq t \leqq T$. First, however, we note that if $\chi_{i}, i=1,2, \cdots$ are each either zero or one, and $M=\sum_{i=1}^{m} \chi_{i}$, then, for any integer $k \leqq m$,

$$
(M)_{k}=M(M-1) \cdots(M-k+1)=\sum^{\prime} \chi_{i_{1}} \cdots \chi_{i_{k}}
$$

where $\sum^{\prime}$ denotes summation over all possible ordered sets of distinct integers $i_{1} \cdots i_{k}$. For $M$ is just the number of non zero $\chi_{i}$, and the right hand side of (10) therefore represents the number of ordered sets of distinct integers $i_{1} \cdots i_{k}$ such that each corresponding $\chi_{i}$ is non zero, taken out of a total of $M$ possible integers $i$ for which $\chi_{i} \neq 0$. But this number is simply $M(M-1) \cdots$ $(M-k+1)$ as required.

Write now $\xi_{i}=\xi\left(T i / 2^{n}\right), i=0,1, \cdots, 2^{n}, n=1,2, \cdots$. Let $\chi_{i}=1$ if $\xi_{i}<u<\xi_{i+1}$, and $\chi_{i}=0$ otherwise. Then if $N_{n}=\sum_{i=1}^{2^{n}} \chi_{i}$ we have $N_{n} \uparrow N$ a.s. ( $A$ detailed proof of this latter statement is given by Ylvisaker [8].) Hence by monotone convergence,

$$
\mathcal{E}\left\{\left(N_{n}\right)_{k}\right\} \rightarrow \mathcal{E}\left\{(N)_{k}\right\} \quad \text { as } n \rightarrow \infty .
$$

Now from (10) we have with $m=2^{n}$,

$$
\mathcal{E}\left\{\left(N_{n}\right)_{k}\right\}=\sum_{i_{1} \cdots i_{k}}^{\prime} P\left\{\chi_{i_{1}}=\chi_{i_{2}}=\cdots=\chi_{i_{k}}={ }^{*} 1\right\} .
$$

We note that no terms for which $\left|i_{r}-i_{s}\right|=1$ for any $r, s$ appear since we cannot have $\chi_{i_{r}}=\chi_{i_{r}+1}=1$. Write $\eta_{i}=2^{n}\left(\xi_{i_{+1}}-\xi_{i}\right) / T$. Then 


$$
\begin{aligned}
P\left\{\chi_{i_{1}}=\chi_{i_{2}}=\cdots=\chi_{i_{k}}=1\right\} & =P\left\{u-2^{-n} T \eta_{i_{r}}<\xi_{i_{r}}<u, r=1 \cdots k\right\} \\
& =\int_{0}^{\infty} \cdots \int_{0}^{\infty} d y_{1} \cdots d y_{k} \int_{u-2^{-n} T y_{1}}^{u} \cdots \\
& \int_{u-2^{-n} T y_{k}}^{u} p_{n, i_{j}}\left(x_{1} \cdots x_{k}, y_{1} \cdots y_{k}\right) d x_{1} \cdots d x_{k},
\end{aligned}
$$

where $p_{n, i_{j}}$ is the joint density for the distribution of $\xi_{i_{1}} \cdots \xi_{i_{k}}, \eta_{i_{1}} \cdots \eta_{i_{k}}$. (That this distribution is non singular follows from the Appendix.) By a change of the $x$-variables in this expression we thus obtain from (12)

$$
\begin{aligned}
& E\left\{\left(N_{n}\right)_{k}\right\}=2^{-k n} T^{k} \sum^{\prime} \int_{0}^{\infty} \cdots \int_{0}^{\infty} d y_{1} \cdots d y_{k} \int_{-y_{1}}^{0} \cdots \\
& \cdot \int_{-y_{k}}^{0} p_{n, \mathbf{i}_{j}}\left(u+2^{-n} T x_{1}, \cdots, u+2^{-n} T x_{k}, y_{1}, \cdots, y_{k}\right) d x_{1} \cdots d x_{k} .
\end{aligned}
$$

Let $A_{n}$ be the subset of $I^{k}$ for which no two of $\left(t_{1} \cdots t_{k}\right)$ are contained in the same or adjacent intervals of the form $\left(r T / 2^{n},(r+1) T / 2^{n}\right)$. Write

$$
\Psi_{n \mathbf{t}}\left(x_{1} \cdots x_{k}, y_{1} \cdots y_{k}\right)=p_{n \mathbf{i}_{j}}\left(x_{1} \cdots x_{k} ; y_{1} \cdots y_{k}\right)
$$

for all $\left(t_{1} \cdots t_{k}\right)$ in $A_{n}$ such that $t_{r}$ lies in the interval $\left(i_{r} T / 2^{n},\left(i_{r}+1\right) T / 2^{n}\right)$ for each $r$, and $\Psi_{n \mathrm{t}}\left(x_{1} \cdots x_{k}, y_{1} \cdots y_{k}\right)=0$ for $\mathrm{t} z A_{n}$. Then (using the remark following Equation (12)), (13) may be rewritten as

$$
\begin{aligned}
& \int_{0}^{T} \cdots \int_{0}^{T} d t_{1} \cdots d t_{k} \int_{0}^{\infty} \cdots \int_{0}^{\infty} d y_{1} \cdots d y_{k} \int_{-y_{1}}^{0} \cdots \\
& \quad \cdot \int_{-y_{k}}^{0} \Psi_{n \mathbf{t}}\left(u+2^{-n} T x_{1}, \cdots, u+2^{-n} T x_{k}, y_{1} \cdots y_{k}\right) d x_{1} \cdots d x_{k} .
\end{aligned}
$$

Let now $\left(t_{1} \cdots t_{k}\right)$ be a fixed point in $A_{n}$. Then $\Psi_{n t}$ is a $2 k$-dimensional normal density function. Suppose that $i_{r} T / 2^{n} \leqq t_{r}<\left(i_{r}+1\right) T / 2^{n}, r=1, \cdots, k$. Then corresponding to the point $t_{r}$ we have the random variables $\xi\left(i_{r} T / 2^{n}\right)$, $\eta\left(i_{r} T / 2^{n}\right)$, yielding the following typical members of the covariance matrix for $\Psi_{n t}$ for example:

$$
\begin{aligned}
\operatorname{var}\left(\xi_{i_{1}}\right) & =r(0), \quad \text { writing } \xi_{2} \text { for } \xi\left(i T / 2^{n}\right), \\
\operatorname{cov}\left(\xi_{i_{1}}, \xi_{i_{2}}\right) & =r_{p}, \quad \text { writing } r_{i} \text { for } r\left(i T / 2^{n}\right), p=i_{1}-i_{2}, \\
\operatorname{cov}\left(\xi_{i_{1}}, \eta_{i_{1}}\right) & =2^{n}\left(r_{1}-r_{0}\right) / T, \\
\operatorname{cov}\left(\xi_{i_{1}}, \eta_{i_{2}}\right) & =2^{n}\left(r_{p+1}-r_{p}\right) / T, \\
\operatorname{var} \eta_{i_{1}} & =2^{2 n+1}\left(r_{0}-r_{1}\right) / T^{2}, \\
\operatorname{cov}\left(\eta_{i_{1}}, \eta_{i_{2}}\right) & =-2^{2 n}\left[r_{p+1}-2 r_{p}+r_{p-1}\right] / T^{2} .
\end{aligned}
$$

For the fixed $t_{1}, t_{2}$ considered $i_{1}, i_{2}, p$ depend on $n$. It is an easy exercise to show that if $\tau=t_{2}-t_{1}$ the above elements converge (in the order given), as $n \rightarrow \infty$, to $r(0), r(\tau), r^{\prime}(0), r^{\prime}(\tau),-r^{\prime \prime}(0),-r^{\prime \prime}(\tau)$, respectively. Similar conclusions hold for the elements corresponding to any pair $t_{i}, t_{j}$. Further, the set $A_{n}$ converges to almost the whole space $I^{k}$. But this means that the integrand in (14) must converge a.e. to $p_{\mathrm{t}}(u, \mathrm{y})$ as $n \rightarrow \infty$ and hence, by Fatou's lemma,

$$
\mathcal{E}\left\{(N)_{k}\right\} \geqq \int_{0}^{T} \cdots \int_{0}^{T} d t_{1} \cdots d t_{k} \int_{0}^{\infty} \cdots \int_{0}^{\infty} y_{1} \cdots y_{k} p_{\mathrm{t}}(u, \mathbf{y}) d y_{1} \cdots d y_{k} .
$$


Combining (9) and (15) we obtain the desired equality and hence the truth of the theorem follows.

3. A case when $M_{k}=+\infty$. Formula (1) was obtained under the condition that $\xi(t)$ have a continuous sample derivative, with probability one. However, this assumption was used in Part A of the proof, but not at all in Part B. Hence if the right hand side of (1) is infinite, the equation is true with both sides infinite. We now give an example of a case where the integral on the right of (1) is infinite, and hence the corresponding moment is infinite.

For this example we take a covariance function of the form

$$
r(\tau)=1-\lambda_{2} \tau^{2} / 2-\tau^{2} / \log |\tau|+o\left(\tau^{2} / \log |\tau|\right) .
$$

That this can be done follows from Theorem 5 of Pitman [5]. In fact we can choose $F(\lambda)$ so that $1-F(\lambda)=1 /\left(2 \lambda^{2} \log ^{2} \lambda\right)$ for all sufficiently large $\lambda$, to give the desired form (16).

Consider now the case $k=2$, and $u=0$. Then one can show by some calculation that

$$
\int_{0}^{\infty} \int_{0}^{\infty} y_{1} y_{2} p_{\mathrm{t}}(0, \mathbf{y}) d y_{1} d y_{2} \sim K|\mathbf{\Lambda}|^{\frac{1}{2}} /\left(1-r^{2}(\tau)\right)
$$

where $K$ denotes some constant and $\mathbf{\Lambda}$ is the covariance matrix for $\xi\left(t_{1}\right), \xi\left(t_{2}\right)$, $\xi^{\prime}\left(t_{1}\right), \xi^{\prime}\left(t_{2}\right), \tau=t_{2}-t_{1}$. But straightforward calculation shows that

$$
|\mathbf{\Lambda}| \sim \lambda_{2} \tau^{2} / \log ^{2}|\tau| \quad \text { as } \quad \tau \rightarrow 0,
$$

and hence

$$
\int_{0}^{\infty} \int_{0}^{\infty} y_{1} y_{2} p_{\mathrm{t}}(0, \mathrm{y}) d y_{1} d y_{2} \sim K /(|\tau| \log |\tau|) \quad \text { as } \quad \tau \rightarrow 0 .
$$

It follows from this that the right hand side of (1) is infinite, in this case.

Finally we note a sufficient condition for $\xi(t)$ to possess a continuous sample derivative, with probability one, is that

$$
r(\tau)=1-\lambda_{2} \tau^{2} / 2+O\left\{\tau^{2} /|\log | \tau||^{a}\right\}
$$

for some $a>1$. This follows from the work of Belayev [1]. In our case $r(\tau)$ given by (16) just fails to satisfy this requirement. Hence it appears that the requirements that $\xi$ have a continuous derivative and that the right hand side of (1) be finite, which are sufficient for $M_{k}$ to be finite and given by (1), are also very close to being necessary for this to be the case.

\section{APPENDIX}

It was stated, in writing down certain density functions that if $t_{1} \cdots t_{k}$ are distinct time points, then

(i) the joint distribution of $\xi\left(t_{1}\right) \cdots \xi\left(t_{k}\right), \xi^{\prime}\left(t_{1}\right) \cdots \xi^{\prime}\left(t_{k}\right)$ is non singular, and

(ii) the joint distribution of $\xi\left(t_{1}\right) \cdots \xi\left(t_{k}\right)$ is non singular.

We shall now prove (i), and hence (ii) will also follow.

Let (as assumed throughout) $F(\lambda)$ have a continuous component and write 
$\mathbf{\Lambda}=\left[\Lambda_{i j}\right]$ for the covariance matrix of $\xi\left(t_{1}\right) \cdots \xi\left(t_{k}\right), \xi^{\prime}\left(t_{1}\right) \cdots \xi^{\prime}\left(t_{k}\right)$. Let $\mathbf{A}=\left[A_{i j}\right]$ denote the covariance matrix of $\xi\left(t_{1}\right) \cdots \xi\left(t_{k}\right), \mathbf{B}=\left[B_{i j}\right]$ that for $\xi^{\prime}\left(t_{1}\right) \cdots \xi^{\prime}\left(t_{k}\right)$, and $\mathbf{C}$ the matrix of "cross" covariances, $C_{i j}=\operatorname{cov}\left(\xi\left(t_{i}\right)\right.$, $\left.\xi^{\prime}\left(t_{j}\right)\right)$. Then

$$
\mathbf{\Lambda}=\left[\begin{array}{ll}
\mathbf{A} & \mathbf{C} \\
\mathbf{C}^{\prime} & \mathbf{B}
\end{array}\right]
$$

Let $\boldsymbol{\theta}$ denote the column vector with elements $\left\{\theta_{1} \cdots \theta_{k}, \phi_{1} \cdots \phi_{k}\right\}$, where $\theta_{i}, \phi_{i}$ are complex numbers which are not all zero. Then we have

$$
\begin{aligned}
& A_{j l}=\int \exp \left[i\left(t_{j}-t_{l}\right) \lambda\right] d F(\lambda), \\
& C_{j l}=-\int i \lambda \exp \left[i\left(t_{j}-t_{l}\right) \lambda\right] d F(\lambda), \\
& B_{i l}=\int \lambda^{2} \exp \left[i\left(t_{j}-t_{l}\right) \lambda\right] d F(\lambda) .
\end{aligned}
$$

From this we see that

$$
\begin{aligned}
\boldsymbol{\theta}^{*} \boldsymbol{\Lambda} \boldsymbol{\theta}=\int\left[\left|\sum_{j} \theta_{j} e^{i t_{j} \lambda}\right|^{2}+\lambda^{2}\left|\sum_{j} \phi_{j} e^{i t t_{j} \lambda}\right|^{2}-i \lambda \sum_{j} \theta_{j} e^{i \lambda t_{j}} \sum_{l} \phi_{l}{ }^{*} e^{-i \lambda t_{l}}\right. \\
\left.+i \lambda \sum_{j} \theta_{j}{ }^{*} e^{-i \lambda t_{j}} \sum_{l} \phi_{l} e^{i \lambda t_{l}}\right] d F(\lambda),
\end{aligned}
$$

in which $a^{*}$ denotes the complex conjugate of a scalar, and the conjugate transpose of a vector. Thus

$$
\boldsymbol{\theta}^{*} \boldsymbol{\Lambda} \boldsymbol{\theta}=\int\left|\sum_{j=1}^{k} \theta_{j} e^{i t_{j} \lambda}+i \lambda \sum_{j=1}^{k} \phi_{j} e^{i t_{j} \lambda}\right|^{2} d F(\lambda) .
$$

Now since the $t_{j}$ are distinct and $\theta_{j}, \phi_{j}$ not all zero it follows that $\sum_{j=1}^{k}\left(\theta_{j} e^{i t_{j} \lambda}+\right.$ $\left.i \lambda \phi_{j} e^{i j_{j} \lambda}\right)$ is a non constant regular function of $\lambda$, viewed as a complex variable, and hence cannot vanish for more than a finite number of values of $\lambda$ in any bounded region. Hence we must have $\boldsymbol{\theta}^{*} \boldsymbol{\Lambda} \boldsymbol{\theta}>0$ since $F(\lambda)$ is not purely discrete. Thus $\boldsymbol{\Lambda}$ is a strictly positive definite matrix and the distribution thus defined is non singular.

The condition that $F(\lambda)$ have a continuous component is a convenient one. However it is (as pointed out by the referee) worth noting that the above proof does apply to spectra which are purely discrete, provided that the set of jumps has at least one finite limiting point.

Finally we note here that the above argument can be easily generalized to include an arbitrary number of derivatives. That is if $F(\lambda)$ has a continuous component and is such that $\xi(t)$ has $n$ sample derivatives $\xi(t) \xi^{\prime}(t) \cdots \xi^{(n)}(t)$, then for any distinct $t_{1}, t_{2}, \cdots, t_{k}$, the joint distribution of $\xi\left(t_{1}\right) \cdots \xi\left(t_{k}\right) \cdots$ $\xi^{(n)}\left(t_{1}\right) \cdots \xi^{(n)}\left(t_{k}\right)$ is non singular.

\section{REFERENCES}

[1] Belayev, YU. K. (1960). Continuity and Hölder's conditions for sample functions of stationary Gaussian processes. Proc. Fourth Berkeley Symp. Math. Statist. Prob. 2 23-33. Univ. of California Press.

[2] Bulinskaya, E. C. (1961). On the mean number of crossings of a level by a stationary Gaussian process. Teor. Veroyatnost. $i$ Primenen 6 474-477. 
[3] Ivanov, V. A. (1960). On the average number of crossings of a level by sample functions of a stochastic process. Teor. Veroyatnost. $i$ Primenen 5 319-323.

[4] Leadbetter, M. R. and Cryer, J. D. (1965). The variance of the number of zeros of a stationary normal processes. Bull. Amer. Math. Soc. 71 561-563.

[5] Pitman, E. J. G. (1960). Some theorems on characteristic functions of probability distributions. Proc. Fourth Berkeley Symp. Math. Statist. Prob. 2 393-402.

[6] Steinberg, H., Schultheiss, P. M., Wogrin, C. A., and Zweig, F. (1955). Short-time frequency measurements of narrow-band random signals by means of zero counting process. J. Appl. Phys. 26 195-201.

[7] Volkonskir, V.A. and Rozanov, Yv. (1961). Some limit theorems for random functions II. Teor. Veroyatnost. $i$ Primenen 6 202-215.

[8] Ylvisaker, N. D. (1965). The expected number of zeros of a stationary Gaussian process. Ann. Math. Statist. 36 1043-1046. 